\title{
Psychopharmacology of Anticonvulsants: Do All Anticonvulsants Have the Same Mechanism of Action?
}

\author{
Stephen M. Stahl, M.D., Ph.D.
}

Issue: Anticonvulsants are not a single therapeutic class, but are composed of multiple distinct subclasses with different mechanisms of action, efficacies, and side effects.

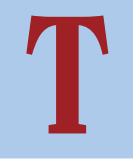

he use of anticonvulsants in psychiatry is rapidly expanding beyond seizure disorders. New uses were originally based on empiric observations, but more recently on an enhanced understanding of how specific psychopharmacologic mechanisms of action might exert therapeutic effects in bipolar disorder, schizophrenia, neuropathic pain, anxiety, and numerous other psychiatric disorders.

\section{Anticonvulsants Do Not All Have} the Same Safety and Efficacy Profiles

All anticonvulsants reduce seizures, but that does not mean they have the same therapeutic actions in conditions other than seizure disorders. For example, some have much better data supporting their use in bipolar disorder (e.g., valproate, carbamazepine, lamotrigine) than do others (e.g., phenytoin, barbiturates, topiramate, gabapentin), ${ }^{1}$ but even agents with evidence of efficacy may act dif-

BRAINSTORMS is a monthly section of The Journal of Clinical Psychiatry aimed at providing updates of novel concepts emerging from the neurosciences that have relevance to the practicing psychiatrist.

From the Neuroscience Education Institute in Carlsbad, Calif., and the Department of Psychiatry at the University of California San Diego.

Reprint requests to: Stephen M. Stahl, M.D. Ph.D., Editor, BRAINSTORMS, Neuroscience Education Institute, 5857 Owens Street, Ste. 102, Carlsbad, CA 92009. ferently on the different phases of that illness. For example, valproate may act most robustly on the manic phase, less robustly on the maintenance phase, and least robustly on the depressed phase, whereas lamotrigine acts most robustly on the maintenance phase, less on the depressed phase, and least on the manic phase. ${ }^{1}$

The same thing can be observed across the anticonvulsants in their actions on pain, with some having little demonstrated efficacy and others differing in their actions on trigeminal neuralgia versus migraine versus neuropathic pain. ${ }^{2}$ As uses broaden, distinctions among the anticonvulsants are sharpening by how they are used in treating various disorders and differences in their safety profiles. ${ }^{1}$

\section{Should Anticonvulsants}

Be Classified by Therapeutic Mechanism?

As clinical and safety differences no longer allow conceptualization of all anticonvulsants as members of a single therapeutic class, neurobiology is beginning to elucidate distinct mechanisms of action that may help explain the clinical differences among the various anticonvulsants, ${ }^{3-5}$ leading to the categorization of anticonvulsants into subtypes according to mechanism of action. One problem with this approach is that some anticonvulsants have well-characterized mechanisms of action, and others do not (Table 1). Nevertheless, data are evolving rapidly, and it is already clear that at least 3 key molecular targets may not only explain how many of the anticonvulsants work but may also serve as the basis for recategorizing them pharmacologically.

\section{Voltage-Gated}

\section{Sodium Channel Antagonists}

Recent advances in ion channel biology have elucidated many subtypes of sodium channels that are opened and closed by changes in voltage across the membranes where they reside. Some, but not all, anticonvulsants directly block the open channel conformation of various sodium channels. These include lamotrigine, carbamazepine, oxcarbazepine, phenytoin, ${ }^{3-5}$ and possibly topiramate (Table 1). Other anticonvulsants such as valproate may have indirect action on sodium channels, perhaps by effects on second messenger systems and enzymes that modulate sodium channels.

Blocking sodium channels leads to reduction of action potentials and thus of seizures and perhaps other symptoms mediated by excessive neurotransmission, such as those in bipolar disorder. Blocking sodium channels also leads to a reduction in release of neurotransmitters such as glutamate and may have other consequences that are still not fully understood. 
Voltage-Gated $\alpha_{2} \delta$-Calcium Channel Subunit Antagonists

Calcium channels comprise another family of ion channels that are opened and closed by changes in voltage across the membranes where they reside. When these channels are located in neuronal membranes at synapses, they are known as $\mathrm{N}$ and $\mathrm{P} / \mathrm{Q}$ channels and regulate neurotransmitter release. Two anticonvulsants, gabapentin and a new agent in clinical testing, pregabalin, act upon calcium channels by binding to their $\alpha_{2} \delta$ subunits to normalize excessively activated calcium channels $^{6}$ (Table 1). Blocking calcium channels in this way reduces the excessive neurotransmitter release that they can trigger.

$\alpha_{2} \delta$-Calcium channel subunit antagonists should not be confused with so-called calcium channel blockers used to treat hypertension. These latter drugs act at a different site on a different calcium channel, namely as antagonists of the pore-forming unit of L-type calcium channels in vascular smooth muscle, lowering blood pressure but not acting as anticonvulsants. $\alpha_{2} \delta-C a l-$ cium channel subunit antagonists on the other hand not only cause anticonvulsant actions, but anxiolytic actions and relief of neuropathic pain.

\section{Direct GABA Modulators}

A unifying hypothesis has been that all anticonvulsants enhance the action of the inhibitory neurotransmitter GABA, but this may be overly simplistic..$^{3-5}$ Although many anticonvulsants raise GABA levels in experimental animals, and several even have "GABA" in their name, only some are direct modulators of GABA, namely tiagabine, benzodiazepines, and barbi-

\section{Take-Home Points}

Anticonvulsants have broad clinical actions in the CNS beyond their ability to reduce seizures, including therapeutic actions in bipolar disorder, neuropathic pain, anxiety, schizophrenia, and, perhaps, other conditions.

Although by definition all anticonvulsants reduce seizures, they have 3 different proposed mechanisms of action: blockade of voltage-gated sodium channels, blockade of voltage-gated calcium channels, and enhancement of GABA actions.

Differences in mechanisms of action may explain differences in therapeutic profiles and side effects of anticonvulsants. Understanding these actions may lead to a more rational drug selection.

Table 1. Hypothetical Mechanisms of Action of Anticonvulsants

\begin{tabular}{lll}
\hline Agent & \multicolumn{1}{c}{ Primary Mechanism } & Secondary Mechanism \\
\hline Valproate & Indirect actions on voltage-gated sodium channels? & Indirectly pro-GABAergic? \\
Carbamazepine & Direct blockade of voltage-gated sodium channels & Indirectly pro-GABAergic? \\
Oxcarbazepine & Direct blockade of voltage-gated sodium channels & Indirectly pro-GABAergic? \\
Topiramate & Direct blockade of voltage-gated sodium channels? & Indirectly pro-GABAergic? \\
Lamotrigine & Direct blockade of voltage-gated sodium channels & Indirectly pro-GABAergic? \\
& & Leads to reduction of glutamate release \\
Phenytoin & Direct blockade of voltage-gated sodium channels & Indirectly pro-GABAergic? \\
Gabapentin & Direct blockade of $\alpha_{2} \delta$ subunits of voltage- & Leads to reduction of release \\
& gated calcium channels & of multiple neurotransmitters \\
Pregabalin & Direct blockade of $\alpha_{2} \delta$ subunits of voltage-gated & Leads to reduction of release \\
& calcium channels & of multiple neurotransmitters \\
Tiagabine & Selective GABA reuptake inhibitor & Raises GABA levels \\
Barbiturates & $\mathrm{GABA}_{\mathrm{A}}$ receptor binding & Enhances GABA actions \\
Benzodiazepines & $\mathrm{GABA}_{\mathrm{A}}$ receptor binding & Enhances GABA actions \\
\hline
\end{tabular}

turates. Other anticonvulsants acting primarily upon voltage-gated sodium or calcium channels may have downstream effects that ultimately change GABA concentrations, but this process remains unclear and in some cases unconvincing compared to the importance of their probable primary action on ion channels.

Tiagabine is a selective inhibitor of the GABA transporter on presynaptic GABA neurons. ${ }^{4,5}$ Thus, GABA levels are immediately and robustly elevated at all GABA receptors, including both $\mathrm{GABA}_{\mathrm{A}}$ and $\mathrm{GABA}_{\mathrm{B}}$ receptors, not only reducing seizures, but perhaps having anxiolytic, pain-reducing, and sleep-enhancing actions. Benzodiazepines and barbiturates, however, act directly upon $\mathrm{GABA}_{\mathrm{A}}$ receptors as allosteric modulators. ${ }^{1,3-5}$ Both cause an enhancement of GABA's inhibitory actions, but only at $\mathrm{GABA}_{\mathrm{A}}$ sites.

\section{Summary}

The mechanisms of action and clinical profiles of anticonvulsants are becoming better characterized. Knowledge of their actions should lead to more rational and effective use of agents in each subclass for specific therapeutic targets.

\section{REFERENCES}

1. Schatzberg AF, Nemeroff CB, eds. Textbook of Psychiatry, 3rd ed. Washington, DC: American Psychiatric Press; 2003

2. Wall PD, Melzack R, eds. Textbook of Pain, 4th ed. London, England: Churchill Livingstone; 1999

3. Manji HK, Bowden CL, Belmaker RH, eds. Bipolar Medications: Mechanisms of Action. Washington, DC: American Psychiatric Press; 2000

4. Sills GJ, Brodie MJ. Epileptic Disord 2001:3:165-172

5. White HS. J Clin Psychiatry 2003;64(suppl 8): $5-8$

6. Gee NS, et al. J Biol Chem 1996;271: 5768-5776 